\title{
Staggered Chiral Perturbation Theory for All-Staggered Heavy-Light Mesons
}

\author{
Javad Komijani* and Claude Bernard \\ Department of Physics, Washington University, St. Louis, MO 63130, USA \\ E-mail: jkomijani@wustl.edu
}

In HISQ simulations by the MILC and Fermilab Lattice collaborations, both the light quarks and the charm quark are staggered. We extend staggered chiral perturbation theory (S $\chi \mathrm{PT})$ to include such all-staggered heavy-light mesons. We assume that the heavy quark action is sufficiently improved that we may take $a m_{Q}<<1$ (where $m_{Q}$ is the heavy quark mass), but also that $m_{Q}>>$ $\Lambda_{Q C D}$ so that a continuum heavy quark expansion is appropriate. Using this $\mathrm{S} \chi \mathrm{PT}$, the leptonic decay constant of the heavy-light meson is calculated at next-to-leading-order. The pattern of taste splittings in the heavy-light meson masses is also investigated.

The 30th International Symposium on Lattice Field Theory - Lattice 2012

June 24-29, 2012

Cairns, Australia

${ }^{*}$ Speaker. 


\section{Introduction}

Heavy-light meson systems provide some of the best ways to test the Standard Model and look for signs of new physics. Lattice QCD provides a means of carrying out non-perturbative calculations from first principles and with controlled errors. In setting up a lattice QCD calculation, a key choice is the form of the lattice action for the quarks.

Staggered fermions [1] are an efficient approach to simulating light quarks. The "highly improved staggered quark" (HISQ) action [2] makes it possible to treat charm quarks with the same action as the light quarks. Thus "all-staggered" simulations of $D$ and $D_{s}$ mesons are now possible [3]. There are several advantages to this all-staggered approach. Probably the most important is that, since heavy and light quarks have the same action, there are partially conserved heavy-light axial and vector currents, which therefore need no renormalization.

Lattice computations often involve an extrapolation in light quark masses to the physical up and down masses, and always require a continuum extrapolation in lattice spacing. A version of chiral perturbation theory $(\chi \mathrm{PT})$ that includes the effects of the discretization errors can help to control these extrapolations. Here, we develop chiral perturbation theory for all-staggered heavylight mesons.

Reference [4] works out a closely related chiral theory for heavy-light mesons with staggered light quarks but non-staggered heavy quarks (for example, Fermilab [5] or NRQCD [6] quarks). In that case, the doubler states of a heavy quark are treated as integrated out, and therefore, heavylight mesons have a single taste degree of freedom associated with the light quark. Here, we need to extend the program developed in Ref. [4] to include staggered heavy quarks with a taste degree of freedom. We assume that the staggered action used (e.g., HISQ) is improved sufficiently that we can treat the heavy quark as "continuum-like," with small corrections from cutoff effects. We refer to this assumption in short-hand as taking $a m_{Q} \ll 1$, where $m_{Q}$ is mass of the heavy quark. We can then use the Symanzik effective theory (SET) [7] to describe the discretization effects on the heavy quarks, as well as on the light quarks.

In the continuum limit, there is an exact $S U(4)$ symmetry acting on tastes; this symmetry is broken at $\mathscr{O}\left(a^{2}\right)$ in the lattice spacing $a$. The corresponding discretization errors in the light-light sector split the masses of mesons with different tastes, which may be understood using staggered chiral perturbation theory (S $\chi \mathrm{PT}$ ) $[8,9]$. For typical values of $a^{2}$, the taste splittings of the light pseudoscalar mesons can be comparable to the masses themselves. Schematically, we say $a^{2} \sim m_{\pi}^{2}$, where appropriate powers of $\Lambda_{Q C D}$ or $\Lambda_{\chi}$ (the chiral scale) are implicitly inserted to match the dimensions in such comparisons. The taste splittings are therefore included in the leading order (LO) light-light Lagrangian, which is of $\mathscr{O}\left(m_{\pi}^{2}\right)$.

For heavy-light mesons composed of staggered quarks, the situation is different. The LO Lagrangian in the continuum is of $\mathscr{O}(k)$, where $k$ is the residual momentum of the heavy-light meson, and we assume that $k \sim m_{\pi}$. Thus, it is reasonable to treat taste violations, which are of $\mathscr{O}\left(a^{2}\right)$, as next-to-leading order (NLO) corrections, and that is what we do here. The LO heavy-light Lagrangian is then taste invariant. This power counting can be checked with HISQ simulations, where the splittings in squared meson masses remain roughly constant as one increases a valence quark mass from the light quark regime to the charm regime [10]. (See also Fig. 1 below.) Therefore the splittings for the masses themselves are much smaller for heavy-light mesons than for light 
mesons. For example, at $a \approx 0.12 \mathrm{fm}$ the measured [10] taste splitting between the root-meansquared (RMS) $D_{s}$ meson and the lightest $D_{s}$ meson is only about $11 \mathrm{MeV}$, while it is about 110 $\mathrm{MeV}$ for the pion $\left(80 \%\right.$ of $m_{\pi}$ ).

\section{The S $\chi$ PT Lagrangian for Heavy-Light Mesons}

We first discuss a heavy-light meson where the light quark has a taste degree of freedom, but the heavy quark does not [4]. Due to the heavy quark spin symmetry in the static limit, the heavy vector and pseudoscalar mesons are incorporated into the following field, which destroys a heavy-light meson,

$$
H_{a}=\frac{1+\psi}{2}\left[\gamma^{\mu} B_{\mu a}^{*}+i \gamma_{5} B_{a}\right]
$$

where $v$ is the meson's velocity, and $a$ is the combined light quark flavor-taste index. Note that we use $B$ to denote a generic pseudoscalar heavy meson and $B^{*}$ to denote the corresponding vector meson, but the practical application of this calculation will, at least in the first instance, be to $D$ mesons.

Now, we assume the heavy quark is also implemented by a staggered fermion. Then we generalize the definition of the annihilation operator of a heavy-light meson as

$$
H_{\alpha a}=\frac{1+\psi}{2}\left[\gamma^{\mu} B_{\mu \alpha a}^{*}+i \gamma_{5} B_{\alpha a}\right]
$$

where $v$ is the meson's velocity, $\alpha$ is the heavy-quark taste index, and $a$ is the combined flavor-taste index of the light quark. The conjugate field creates a heavy-light meson

$$
\bar{H}_{a \alpha} \equiv \gamma_{0} H_{a \alpha}^{\dagger} \gamma_{0}=\left[\gamma^{\mu} B_{\mu a \alpha}^{\dagger *}+i \gamma_{5} B_{a \alpha}^{\dagger}\right] \frac{1+\psi}{2} .
$$

So far $H$ is treated as a $4 \times 4 n$ matrix in the taste and the flavor space of quarks. Instead of attaching separate indices to the tastes of the light and the heavy quarks, one might use just one index as the taste of the meson. Then, one treats $H$ as a $n$-component vector in the flavor space of the light quark, while each element $\left(H_{i}\right)$ is a $4 \times 4$ matrix in the taste space of the meson. To implement this approach, the light flavor-taste index is first traded for a pair of indices representing flavor and taste separately. We use Latin indices in the middle of the alphabet $(i, j, \ldots)$ as pure flavor indices. We can then combine the tastes of the heavy and the light quarks and use an index such as $\Xi$ (where $\Xi=1, \ldots 16$ ) as the taste of the meson. Therefore, the $i$ th element of the field destroying a heavy-light meson in the light flavor space can be represented by $H_{i}=\sum_{\Xi=1}^{16} H_{i \Xi} T_{\Xi}$ and its conjugate by $\bar{H}_{i}=\sum_{\Xi=1}^{16} \bar{H}_{i \Xi} T_{\Xi}$, with the Hermitian taste generators $T_{\Xi} \in\left\{\xi_{5}, i \xi_{\mu 5}, i \xi_{\mu v}, \xi_{\mu}, \xi_{I}\right\}$.

The leading-order staggered version of the heavy-light meson Lagrangian then looks the same as in the case with only light staggered fields, or indeed in standard continuum $\chi$ PT, with the only change being the extra taste degrees of freedom in the fields. The LO chiral Lagrangian involving the heavy-light meson fields is:

$$
\mathscr{L}_{1}=-i \operatorname{Tr}(\bar{H} H v \cdot \overleftarrow{D})+g_{\pi} \operatorname{Tr}\left(\bar{H} H \gamma^{\mu} \gamma_{5} \mathbb{A}_{\mu}\right)
$$

where $(\bar{H} H)_{a b} \equiv \bar{H}_{a \alpha} H_{\alpha b}$, and Tr, here and below, means the complete trace over flavor and taste indices and, where relevant, Dirac indices. This Lagrangian has separate $S U(4)$ taste symmetries on the heavy and light quarks, as well as spin symmetry of the heavy quark. 
To derive the heavy-light decay constants, we also will need the chiral representative of the axial heavy-light current. Alternatively, one can work with the left-handed current. The left-handed current that destroys a heavy-light meson of taste $\Xi$ and light flavor $i$ is $j^{\mu, i \Xi}$, which at LO takes the form

$$
j_{\mathrm{LO}}^{\mu, i \Xi}=\frac{\kappa}{2} \operatorname{tr}_{D, t}\left(T_{\Xi} \gamma^{\mu}\left(1-\gamma_{5}\right) H \sigma^{\dagger} \lambda^{(i)}\right)
$$

where $\operatorname{tr}_{D, t}$ is a trace over Dirac and taste indices, and $\lambda^{(i)}$ is a constant row vector that fixes the flavor of the light quark: $\left(\lambda^{(i)}\right)_{j}=\delta_{i j}$. The decay constant $f_{B_{i \Xi}}$ is defined by the matrix element

$$
\left\langle 0\left|j^{\mu, i^{\prime} \Xi^{\prime}}\right| B_{i \Xi}(v)\right\rangle=i f_{B_{i \Xi}} \sqrt{m_{B_{i \Xi}}} v^{\mu} \delta_{\Xi \Xi^{\prime}} \delta_{i i^{\prime}}
$$

where the state $\left|B_{i \Xi}(v)\right\rangle$ is normalized non-relativistically, corresponding to our non-relativistically normalized heavy meson field $B$. At LO in the heavy-light chiral theory, $j_{\mathrm{LO}}^{\mu, i^{\prime} \Xi^{\prime}}=i \kappa v^{\mu} B_{i^{\prime} \Xi^{\prime}}$, which gives $f_{B_{i \Xi}}^{\mathrm{LO}}=\kappa / \sqrt{m_{B_{i \Xi}}}$.

To go beyond leading order, we must encode the discretization errors in the chiral theory. To do this we consider a series of effective field theories. Assuming $a m_{Q}<<1$, we can derive a Symanzik effective theory (SET) from the staggered lattice Lagrangian. One can then use the fact that $m_{Q}$ is large compared to $\Lambda_{Q C D}$ to organize heavy quark effects with heavy quark effective theory (HQET). Finally, when residual momenta and light quark masses are small compared to the chiral scale $\Lambda_{\chi} \sim 1 \mathrm{GeV}$, the physics of light-light and heavy-light mesons may be described by a chiral effective theory. Here, we assume the power counting $p_{\pi}^{2} \sim m_{\pi}^{2} \sim m_{q} \sim a^{2}$ for the light mesons as in Ref. [4].

There are three different types of 4-quark operators in the SET at order $a^{2}$ :

$$
\begin{aligned}
a^{2} \mathscr{O}_{s s^{\prime} t t^{\prime}} & =c_{1} a^{2} \bar{q}_{l}\left(\gamma_{s} \otimes \xi_{t}\right) q_{l} \bar{q}_{l^{\prime}}\left(\gamma_{s^{\prime}} \otimes \xi_{t^{\prime}}\right) q_{l^{\prime}} \\
& +c_{2} a^{2} \bar{q}_{l}\left(\gamma_{s} \otimes \xi_{t}\right) q_{l} \bar{q}_{h}\left(\gamma_{s^{\prime}} \otimes \xi_{t^{\prime}}\right) q_{h} \\
& +c_{3} a^{2} \bar{q}_{h}\left(\gamma_{s} \otimes \xi_{t}\right) q_{h} \bar{q}_{h^{\prime}}\left(\gamma_{s^{\prime}} \otimes \xi_{t^{\prime}}\right) q_{h^{\prime}}
\end{aligned}
$$

where $q_{l}$ and $q_{h}$ are the light and heavy quark fields, and $\gamma_{s}$ and $\xi_{t}$ are any of the 16 spin or taste matrices, respectively. The operators listed in Eq. (2.7) are generic, and stand for a linear combination of all operators with the same heavy and light quark structure. The first type of operator has only light quarks and hence respects the heavy taste symmetry. Its contributions to the NLO chiral Lagrangian are thus essentially the same as those that appear when the heavy quark has no taste, i.e., those of Ref. [4]. Although the third type breaks the heavy taste symmetry, it contributes only in trivial ways to the heavy-light Lagrangian, which by definition has only two meson fields and does not describe heavy-light meson scattering. New chiral terms arise only from from the second type of operator, which has both heavy and light fields.

Using a spurion analysis, we find that the operators of the second type lead to the following two contributions to the NLO Lagrangian:

$$
\begin{aligned}
\mathscr{L}_{2, a^{2}}^{A 2}= & K_{A 1} a^{2} \operatorname{Tr}\left(\bar{H} \xi_{5} H \xi_{5}\right)+K_{A 2} a^{2} \operatorname{Tr}\left(\bar{H} \xi_{\mu} H \xi_{\mu}\right)+K_{A 3} a^{2} \operatorname{Tr}\left(\bar{H} \xi_{5 \mu} H \xi_{\mu 5}\right) \\
& +K_{A 4} a^{2} \operatorname{Tr}\left(\bar{H} \xi_{\mu v} H \xi_{v \mu}\right)+K_{A 5} a^{2} \operatorname{Tr}\left(\bar{H} \gamma_{5 \mu} H \gamma^{\mu 5}\right) \\
& +K_{A 6} a^{2} \operatorname{Tr}\left(\bar{H} \gamma_{5 \mu} \xi_{5} H \gamma^{\mu 5} \xi_{5}\right)+K_{A 7} a^{2} \operatorname{Tr}\left(\bar{H} \gamma_{\mu \nu} \xi_{\lambda} H \gamma^{v \mu} \xi_{\lambda}\right) \\
& +K_{A 8} a^{2} \operatorname{Tr}\left(\bar{H} \gamma_{\mu \nu} \xi_{5 \lambda} H \gamma^{\nu \mu} \xi_{\lambda 5}\right)+K_{A 9} a^{2} \operatorname{Tr}\left(\bar{H} \gamma_{5 \mu} \xi_{v \lambda} H \gamma^{\mu 5} \xi_{\lambda v}\right)
\end{aligned}
$$


and

$$
\begin{aligned}
\mathscr{L}_{2, a^{2}}^{B 2}= & \sum_{\mu}\left[K_{B 1} a^{2} \operatorname{Tr}\left(\bar{H} \gamma_{v \mu} \xi_{\mu} H \gamma^{\mu v} \xi_{\mu}\right)+K_{B 2} a^{2} \operatorname{Tr}\left(\bar{H} \gamma_{v \mu} \xi_{5 \mu} H \gamma^{\mu v} \xi_{\mu 5}\right)\right. \\
& \left.+K_{B 3} a^{2} v^{\mu} v_{\mu} \operatorname{Tr}\left(\bar{H} \xi_{v \mu} H \xi_{\mu v}\right)+K_{B 4} a^{2} \operatorname{Tr}\left(\bar{H} \gamma_{5 \mu} \xi_{v \mu} H \gamma^{\mu 5} \xi_{\mu v}\right)\right],
\end{aligned}
$$

where $\mathscr{L}_{2, a^{2}}^{A 2}$ (the "type-A" contribution) results from operators that are invariant over the full Euclidean space-time rotation group, $S O(4)$, as well as a corresponding $S O(4)$ of taste, and $\mathscr{L}_{2, a^{2}}^{B 2}$ (the "type-B" contribution) results from operators that couple spin and taste and break these $S O(4)$ symmetries.

\section{Results and Conclusions}

The $\mathscr{O}\left(a^{2}\right)$ contributions to the Lagrangian, $\mathscr{L}_{2, a^{2}}^{A 2}$ and $\mathscr{L}_{2, a^{2}}^{B 2}$, give different NLO mass corrections to different tastes of the heavy-light mesons. The type-A terms split the masses into the five $S O(4)$ taste multiplets, labeled by I, V, T, A, and P. The type-B terms split these representations and give different masses to the time and spatial components, such as $\xi_{0}$ and $\xi_{i}$ for the vector taste (V) representation. Note that no splitting, either between $S O(4)$ multiplets, or within the multiplets, comes from the one-loop diagrams. This can be proved using the exact $S U$ (4) heavy-quark taste symmetry of the LO Lagrangian and the discrete taste symmetry of the light quarks, which corresponds to the shift symmetry in the staggered action [4].

Based on the pattern of splittings seen at LO in light mesons, the 4-quark operators with the taste structure $\xi_{\mu 5}$ and spin $I, \gamma_{5}$, or $\gamma_{\mu v}$ appear to be dominant. These operators contribute to the $C_{4}$ chiral operator [9], which gives the characteristic P, A, T, V, I (lowest-to-highest) ordering of squared masses, with roughly equal spacing. Note that, for light mesons, only type-A 4-quark operators are relevant to LO, because type-B operators have no chiral representatives until NLO [8]. Here the corresponding type-A 4-quark operators give rise to the chiral operators $K_{A 3} a^{2} \operatorname{Tr}\left(\bar{H} \xi_{5 \mu} H \xi_{\mu 5}\right)$ and $K_{A 8} a^{2} \operatorname{Tr}\left(\bar{H} \gamma_{\mu \nu} \xi_{5 \lambda} H \gamma^{\nu \mu} \xi_{\lambda 5}\right)$. These produce the same equal-spacing pattern for the $S O(4)$ taste representations of heavy-light mesons: See Table 1. For type-B operators, one might guess that the taste $\xi_{\mu 5}$ and spin $\gamma_{\mu \nu}$ 4-quark operator would be dominant, since it is the only type-B operator that has the same spin and taste as one of the dominant type-A operators. This 4-quark operator gives rise to the chiral operator $\sum_{\mu} K_{B 2} a^{2} \operatorname{Tr}\left(\bar{H} \gamma_{v \mu} \xi_{5 \mu} H \gamma^{\mu v} \xi_{\mu 5}\right)$. Table 2 shows the pattern of mass splitting that stems from this type-B operator.

The patterns given in Tables 1 and 2 are qualitatively present in the MILC data, shown in Fig. 1. Note in particular the "sc" case, for which the errors are small enough that the pattern of $S O(4)$ breaking for heavy-light mesons is clear. It is non-trivial that the time component of taste is higher than the space component in two cases $\left(\xi_{0} v s . \xi_{i}\right.$ and $\left.\xi_{i 0} v s . \xi_{i j}\right)$ but not in the third case ( $\left.\xi_{05} v s . \xi_{i 5}\right)$, just as in Table 2. Although the chiral theory is not applicable to the "cc" case, it is interesting to see that the structure that would correspond to the dominant type-B operator gets particularly strong there, with near degeneracies of between members of different $S O(4)$ multiplets, in particular $\xi_{0}$ and $I$, or $\xi_{i 0}$ and $\xi_{i}$.

We can also calculate the decay constant of the heavy-light meson to NLO. At one loop, we express the decay constant as

$$
f_{B_{x \Xi}}=f_{B_{x}}^{\mathrm{LO}}\left(1+\frac{1}{16 \pi^{2} f^{2}} \delta f_{B_{x}}+\text { analytic terms }\right) .
$$


Table 1: Taste splittings due to the apparently dominant type-A operators.

\begin{tabular}{|c||c|c|c|c|c|}
\hline & $\triangle_{m_{Q}}\left(\xi_{5}\right)$ & $\triangle_{m_{Q}}\left(\xi_{5 \mu}\right)$ & $\triangle_{m_{Q}}\left(\xi_{\mu v}\right)$ & $\triangle_{m_{Q}}\left(\xi_{\mu}\right)$ & $\triangle_{m_{Q}}(I)$ \\
\hline \hline $8 a^{2}\left(K_{A 3}+4 K_{A 8}\right)$ & -4 & -2 & 0 & +2 & +4 \\
\hline
\end{tabular}

Table 2: Taste splittings due to the apparently dominant type-B operator.

\begin{tabular}{|c||c|cc|cc|cc|c|}
\hline & $\triangle_{m_{Q}}\left(\xi_{5}\right)$ & $\triangle_{m_{Q}}\left(\xi_{05}\right)$ & $\triangle_{m_{Q}}\left(\xi_{i 5}\right)$ & $\triangle_{m_{Q}}\left(\xi_{i j}\right)$ & $\triangle_{m_{Q}}\left(\xi_{i 0}\right)$ & $\triangle_{m_{Q}}\left(\xi_{i}\right)$ & $\triangle_{m_{Q}}\left(\xi_{0}\right)$ & $\triangle_{m_{Q}}(I)$ \\
\hline \hline $8 a^{2} K_{B 2}$ & -6 & -6 & -2 & -2 & +2 & +2 & +6 & +6 \\
\hline
\end{tabular}

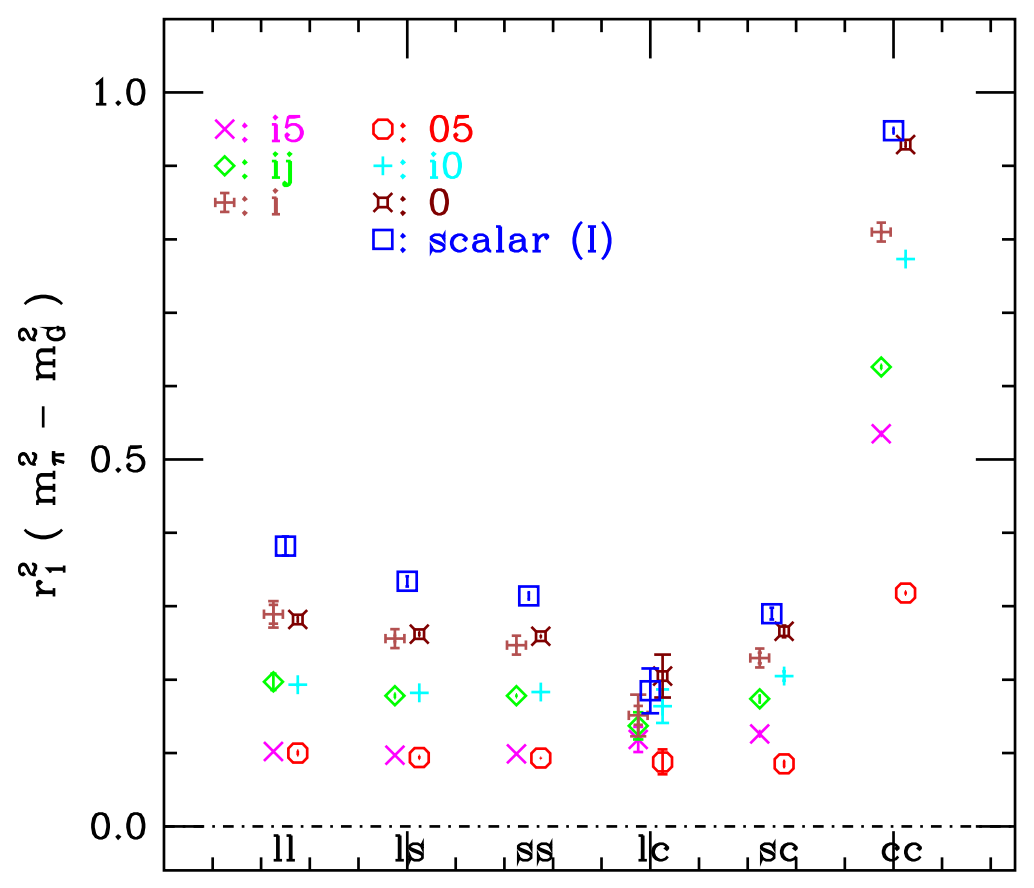

Figure 1: MILC HISQ ensemble at $a \approx 0.15 \mathrm{fm}$ and $m_{l}=0.2 m_{s}$ [10]. Squared mass splitting between pions of different tastes and the Goldstone pion in units of $r_{1}$. The types of quarks in the mesons are shown on the abscissa: $1, \mathrm{~s}$, and c stand for light (u,d), strange, and charm quarks, respectively.

The lowest order term $f_{B_{x}}^{\mathrm{LO}}$ depends only on the light valence flavor as $f_{B_{x}}^{\mathrm{LO}}=\kappa / \sqrt{m_{B_{x}}}$ because the taste splittings of heavy-light masses appear at NLO. We can divide the terms contributing to the decay constant at NLO into two parts. The first part comes from the operators which obey the $S U(4)$ taste symmetry of the heavy quarks, while the second stems from those breaking the heavy taste symmetry. The first part contributes both to the analytic terms and to the chiral logarithms, $\delta f_{B_{x}}$ in Eq. (3.1). It is independent of the taste of the meson, and indeed is the same as the result in Ref. [4], because it obeys both the residual discrete taste symmetry of the light quarks and the $S U(4)$ taste symmetry of the heavy quarks at LO. The second part contributes only to the analytic 
terms in Eq. (3.1), which depend on the taste of the meson.

We find the decay constant of the heavy-light meson in the partially quenched case is:

$$
\begin{aligned}
\left(\frac{f_{B_{x \Xi}}}{f_{B_{x}}^{\mathrm{LO}}}\right)_{1+1+1}= & 1+\frac{1}{16 \pi^{2} f^{2}} \frac{1+3 g_{\pi}^{2}}{2}\left\{-\frac{1}{16} \sum_{f, \Xi^{\prime}} \ell\left(m_{x f, \Xi^{\prime}}^{2}\right)\right. \\
& -\frac{1}{3} \sum_{j \in \mathscr{M}_{I}^{(3, x)}} \frac{\partial}{\partial m_{X, I}^{2}}\left[R_{j}^{[3,3]}\left(\mathscr{M}_{I}^{(3, x)} ; \mu_{I}^{(3)}\right) \ell\left(m_{j}^{2}\right)\right] \\
& \left.-\left(a^{2} \delta_{V}^{\prime} \sum_{j \in \mathscr{M}_{V}^{(4, x)}} \frac{\partial}{\partial m_{X, V}^{2}}\left[R_{j}^{[4,3]}\left(\mathscr{M}_{V}^{(4, x)} ; \mu_{V}^{(3)}\right) \ell\left(m_{j}^{2}\right)\right]+[V \rightarrow A]\right)\right\} \\
& +c_{s}\left(m_{u}+m_{d}+m_{s}\right)+c_{v} m_{x}+c_{a, \Xi} a^{2},
\end{aligned}
$$

where $x$ is the valence flavor, $\Xi$ is the valence taste, $f$ runs over the three sea quarks $u, d$, and $s, \Xi^{\prime}$ runs over the 16 meson tastes, and other notation is explained in Ref. [4]. In Eq. (3.2), $c_{a, \Xi}$ is the only coefficient which depends on the taste of the heavy meson.

In summary, we have generalized the chiral Lagrangian of a heavy-light meson to the case where both heavy and light quarks have taste degrees of freedom. We have obtained the NLO chiral Lagrangian, which breaks the $S U(4)$ heavy taste symmetry. Moreover we have derived the NLO decay constants and the taste splittings of the heavy-light masses, and have used them to understand, qualitatively, the pattern of splittings seen in the heavy-light HISQ data.

We thank our colleagues in MILC for Figure 1, and Andreas Kronfeld for helpful discussions on the use of effective theories for this problem. Our work was supported in part by the U.S. Department of Energy under Grant DE-FG02-91ER40628.

\section{References}

[1] J. B. Kogut and L. Susskind, Phys. Rev. D 11, 395 (1975). T. Banks et al., Phys. Rev. D 15, 1111 (1977); L. Susskind, Phys. Rev. D 16, 3031 (1977).

[2] E. Follana et al. [HPQCD Collaboration], Phys. Rev. D 75, 054502 (2007) [hep-lat/0610092].

[3] E. Follana, C. T. H. Davies, G. P. Lepage and J. Shigemitsu [HPQCD Collaboration and UKQCD Collaboration], Phys. Rev. Lett. 100, 062002 (2008) [arXiv:0706.1726]; J. Bailey et al. [MILC and Fermilab Lattice Collaborations], POS (LAT2012) 159.

[4] C. Aubin and C. Bernard, Phys. Rev. D73 014515 (2006) [arXiv:hep-lat/0510088v3].

[5] A. X. El-Khadra, A. S. Kronfeld and P. B. Mackenzie, Phys. Rev. D 55, 3933 (1997) [arXiv:hep-lat/9604004].

[6] G. P. Lepage and B. A. Thacker, Nucl. Phys. Proc. Suppl. 4, 199 (1988); B. A. Thacker and G. P. Lepage, Phys. Rev. D 43, 196 (1991).

[7] K. Symanzik, Nucl. Phys. B 226, 187 (187).

[8] W. Lee and S. Sharpe Phys. Rev. D60, 114503 (1999) [arXiv:hep-lat/9905023].

[9] C. Aubin and C. Bernard, Phys. Rev. D 68, 034014 (2003) [arXiv:hep-lat/0304014] and Phys. Rev. D 68, 074011 (2003) [arXiv:hep-lat/0306026].

[10] A. Bazavov et al.[MILC Collaboration], in preparation. 